\title{
Thermoelectric Power Measurement of Gel Grown Strontium Oxalate Crystals
}

\author{
Paresh Vasantlal Dalal ${ }^{1}$ \\ ${ }^{I}$ (Research Lab, Department of Physics, Shri Vitthalrao Shankarrao Naik Arts, Commerce and Science College, \\ Raver-425508, India
}

\begin{abstract}
Thermo Electric Power (TEP) measurement of gel grown strontium oxalate crystals was carried out. Different parameters such as Fermi energy and mode of scattering were calculated. To calculate Fermi energy and scattering parameter of a material, a graph of Seebeck coefficient $(S)$ versus reciprocal of temperature difference (1/4T) is plotted. The slope of the graph is $26.92 \mathrm{mV}$ and intercept is $0.179 \mathrm{mV} / \mathrm{K}$, and hence Fermi energy, $E_{F}=0.027 \mathrm{eV}$. Scattering parameter has calculated 0.425. The experimental value obtained for $A=$ 2.075 is in well agreement to conclude that the conduction of heat in the material may be due to the lattice or phonons and can be associated with lattice or phonon scattering.
\end{abstract}

Keywords: Strontium oxalate, Gel technique, TEP, Fermi energy, Mode of scattering

\section{Introduction}

Energy crisis over the world has emphasized on our scientist to think on small power generation sources like Thermoelectric power (TEP), which is environment friendly and could be played vital role at large scale.TEP has attracted for its applications in power generation and in designing a portable refrigerator [1]. Many scientist have made use of semiconducting material [2] with a maximum operating temperature of $200{ }^{\circ} \mathrm{C}$ [3]. Narrow gap semiconductors and its alloys are found to be the most efficient thermo-electric materials [4][5], because of their high Seebeck coefficient and therefore they have high demand in electronics field. Insulators exhibit large TEP, as they have extremely high resistance; therefore conduction take place by several mechanisms such as thermal or Schottky emission, tunnelling, Poole-Frenkel effect, field emission, space charge limited conduction etc.[6]. Measurement of thermoelectric power has distinctive advantages over other methods because measured thermoelectric voltage is directly related to the carrier concentration [7]. Many researchers have reported thermoelectric power measurement of semiconducting materials [8]-[9], and some on organic materials [10]-[17]. However, there are no such studies are reported on oxalate material.

Looking at the importance of the thermoelectric power (TEP), author of this paper has therefore carried out a systematic investigation of gel grown strontium oxalate crystals. Different parameters such as Fermi energy and mode of scattering were also calculated.

\section{Experimental}

Simple, inexpensive and suitable gel method [18-20] was used to grow strontium oxalate crystals at ambient temperature [21]. These gel grown strontium oxalate crystals were then used for the thermoelectric power studies. Thermo electric power of strontium oxalate single crystal was measured on an instrument assembled in the Physics Research Lab, Shri V. S. Naik Arts, Commerce and Science College, Raver. (Maharashtra). A single crystal of strontium oxalate was placed in the instrument at a proper position and made all equipment ready for working. One end of the crystal was heated with the heating device upto certain fixed temperature. The temperature difference occurred between two ends and corresponding developed e.m.f. were recorded.

\section{Results And Discussion}

Employing electrical heating device to one end of a crystal produces a temperature gradient, $(\Delta \mathrm{T})$ between hot and cold junctions of a material and give rise to an e.m.f. The developed thermo e.m.f. $(\Delta \mathrm{E})$ and temperatures of hot and cold junction are recorded and used to calculate thermo electric power or Seebeck coefficient ' $\mathrm{S}$ '. This developed e.m.f. is directly proportional to $\Delta \mathrm{T}$. The temperature difference, $\Delta \mathrm{T}$ between two ends of a crystal is ranging within a limit of 9 to $12 \mathrm{~K}$.Thus, Seebeck coefficients ' $\mathrm{S}$ ' at different temperatures were calculated by using the equation:

$$
\mathrm{S}=(\Delta \mathrm{E} / \Delta \mathrm{T})
$$

The variation of thermo electric power, or Seebeck coefficient $(\mathrm{S})$ and reciprocal of absolute temperature difference $(1 / \Delta T)$ is shown in Fig. 1. 


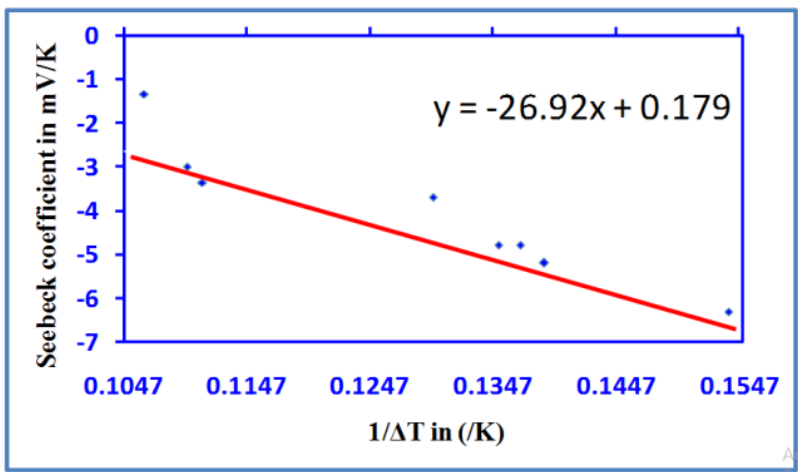

Figure 1: Plot of Seebeck coefficient versus Reciprocal of Temperature

This graph (Fig. 1) was used to calculate Fermi energy $\left(\mathbf{E}_{\mathrm{F}}\right)$ and scattering parameter $(\alpha)$ as-

\begin{tabular}{|c|c|c|c|}
\hline \multirow{5}{*}{\multicolumn{2}{|c|}{ Fermi energy $\left(\mathbf{E}_{\mathbf{F}}\right)$}} & $=$ & (slope $\mathrm{X}$ charge on electron in joule) \\
\hline & & $=$ & $26.92 \times 1.6 \times 10^{-19}$ Joule \\
\hline & & $=$ & $26.92 \mathrm{meV}$ \\
\hline & & $=$ & $0.02692 \mathrm{eV}$ \\
\hline & & $=$ & $0.027 \mathrm{eV}$ \\
\hline \multirow{2}{*}{\multicolumn{2}{|c|}{ Constant A }} & $=$ & (intercept $\mathrm{x}$ e)/K \\
\hline & & $=$ & $\frac{0.179 \times 1.6 \times 10^{-25} \times 10^{-3}}{1.38 \times 10^{-25}}$ \\
\hline \multirow{3}{*}{ Scattering parameter } & A & $=$ & 2.075 \\
\hline & $\alpha_{\mathrm{s}}$ & $=$ & $5 / 2-2.075$ \\
\hline & & $=$ & 0.425 \\
\hline
\end{tabular}

The Fermi energy, ' $\mathrm{E}_{\mathrm{F}}$ ' and constant ' $\mathrm{A}$ ' were obtained from the graph and used to find mode of scattering responsible for the conduction of heat through the material. The constant, $\mathrm{A}=2.075$ obtained from the graph is suggesting (TABLE 1) that the conduction of heat through the strontium oxalate crystal might be due to the lattice or phonons scattering [6].

Table 1: Values of A and their corresponding Mode of scattering

\begin{tabular}{ll}
\hline Scattering mode & Constant A \\
\hline Ionized impurity & 4 \\
Piezoelectric (ionic lattice) & 3 \\
Grain boundary & - \\
Lattice or phonons & 2 \\
Vibrations at & 1 \\
Constant frequency & 0.5 \\
Ionic lattice & 2.5 \\
Degenerate system & - \\
\hline
\end{tabular}

\section{Conclusion}

In the present study, gel grown strontium oxalate single crystal was characterized by Thermo electric power (TEP) measurement. From the above studies, following points are observed:

1. Calculated Fermi energy $E_{\mathrm{F}}=0.027 \mathrm{eV}$

2. Calculated Scattering parameter $\alpha_{\mathrm{s}}=0.425$

3. It is concluded that the conduction of heat in the material may be due to lattice or phonon

4. And, can be associated with lattice or phonon scattering.

\section{Acknowledgements}

The corresponding author is thankful to INSA, New Delhi for awarding 'Visiting Fellowship'. Thankful to the University Grants Commission, New-Delhi for sanctioning Minor Research Project and also thankful to The, Principal, Shri Vitthalrao Shankarrao Naik for providing laboratory facilities.

\section{References}

[1] G. Mahan, Good Thermo electrics, Solid State Physics, Vol. 51, 1998, pp. 81-157.

[2] H. Goldsmid, J. Cochrane, Solar thermoelectric generators. Proceedings of the 4th IEEE International Conference on Thermoelectric Energy Conversion, Arlington, TX, USA, 1982

[3] F. Rosi, Thermoelectricity and thermoelectric power generation, Solid-State Electron., Vol.11,1968, pp. 833-848.

[4] C. Wood, Materials for thermoelectric energy conversion, Rep. Prog. Phys., Vol. 51, 1988, pp.459-539. 
[5] H. Goldsmid, and R. Douglas, The use of semiconductors in thermoelectric refrigeration, Brit. J. Appl. Phys., Vol. 5, 1954 , pp. 386.

[6] A. Goswami, Thin film Fundamentals (Age International (P) Limited, New Delhi, 1996)

[7] L. Brixner, X-ray study and thermoelectric properties of the $\mathrm{W}_{\mathrm{x}} \mathrm{Ta}_{1-\mathrm{x}} \mathrm{Se}_{2}$, J. Electrochem Soc, Vol.110, 1963, pp. 289.

[8] T. Calliat, J. Fileurial, Preparation and thermoelectric properties of semiconducting $\mathrm{Zn}_{4} \mathrm{Sb}_{3}$, J. Phys Chem Solid, Vol. 58(7), 1997, pp. 1119.

[9] V. Static, A. Pierre, T. Etsall, and R. Mikula, Preparation of tungsten sulfides by sol-gel processing, J Non-Cryst Solids, Vo. 220,1997, pp. 58.

[10] Q. Wei, M. Mukaida, K. Kirihara, Y. Naitoh,, T. Ishida, Thermoelectric power enhancement of PEDOT:PSS in high-humidity conditions, Appl. Phys. Express, Vol. 7, 2014, 031601

[11] Q. Wei, M. Mukaida, K. Kirihara, T. Ishida, Experimental studies on the anisotropic thermoelectric properties of conducting polymer films, ACS Macro Lett., Vol. 3, 2014, pp. 948-952.

[12] K. Chang, M. Jeng, C. Yang, Y. Chou, S. Wu, M. Thomas, Y. Peng, The thermoelectric performance of poly(3,4ethylenedioxythiophene)/poly(4styrenesulfonate) thin films, J. Electron. Mater., Vol. 38, 2009, pp. 1182-1188.

[13] B. Zhang, J. Sun, H. Katz, F. Fang, R. Opila, Promising thermoelectric properties of commercial PEDOT:PSS materials and their Bi2Te3 powder composites, ACS Appl. Mater. Interfaces, Vol. 2, 2010, pp. 3170-3178.

[14] G. Kim, L. Shao, K. Zhang, K. Pipe, Engineered doping of organic semiconductors for enhanced thermoelectric efficiency, Nat. Mater. Vol. 12, 2013, pp. 719-723.

[15] H. Park, S. Lee, F. Kim, H. Choi, I. Cheong, Enhanced thermoelectric properties of PEDOT:PSS nano films by a chemical dedoping process, J. Mater. Chem. A, Vol.2, 2014, pp. 6532-6539.

[16] B. McGrail, A. Sehirlioglu, E. Pentzer, Polymer composites for thermoelectric applications, Angew. Chem. Int. Ed., Vol. 54, 2015, pp. 1710-1723.

[17] A. Yoshida, N. Toshima, Gold nanoparticle and gold nanorod embedded PEDOT:PSS thin films as organic thermoelectric materials, J. Electron. Mater. Vol. 43, 2014, pp. 1492-1497.

[18] I. Patel, D. Vaghela, K. Rathod, V. Tandel, Study of thermal properties of ZTS crystal grown by gel technique, IJIRAS, Vol. 4, 2017, pp. 210-212.

[19] P. Dalal, K. Saraf, Growth and study of barium oxalate single crystals in agar gel, Bull. Mater. Sci., Vol. 29, 2006, pp. 421-425

[20] P. Dalal, K. Saraf, S. Shah, Growth of Barium Oxalate Crystals in Agar-Agar Gel and Their Characterization, Crystal Research and Technology, Vol. 44, 2009, pp. 36-42.

[21] P. Dalal, K. Saraf, Growth of strontium oxalate crystals in agar-agar gel, Bull. Mater. Sci., Vol. 34, 2011, pp. 377-381 